\title{
VARIOUS WEATHER CONDITIONS USING DARK CHANNEL AND FUZZY LOGIC FOR REAL TIME VIDEOS
}

\author{
T.Saikumar ${ }^{1}$, Rama Swamy Komma ${ }^{2}$ \\ ${ }^{I}$ Sr, Asst. Professor, Department of ECE,CMRTC, Hyderabad, T.S, India \\ ${ }^{2}$ B.Tech (Scholar), Department of ECE, CMRTC, Hyderabad, T.S, India
}

\begin{abstract}
Rain, fog and haze are natural phenomena that fade scenes, limit the visibility range, and cause shifts in colors. These phenomena also play a decisive role in determining the degree of reliability of many kinds of outdoor applications, such as aerial and satellite imaging, surveillance, and driver assistance systems. Thus, removing their effects from images/videos is very crucial. Due to its mathematically ill posed nature, enhancement process of rain, fog, and haze plagued images/videos is highly challenging. In this paper, we propose a fast yet robust technique to enhance the visibility of video frames using the dark channel prior combined with fuzzy logic-based technique. The dark channel prior is a statistical regularity of outdoor haze-free images based on the observation that most local patches in the haze-free images contain pixels which are dark in at least one color channel, where the fuzzy logic-based technique is used to map an input space to an output space using a collection of fuzzy membership functions and rules to decide softly in case of uncertainties. The combination of the dark channel and the fuzzy logic-based technique will produce high quality haze-free images in real-time. Furthermore, it will be combined with rules derived from the stable atmospheric scattering model and will yield a fast yet high quality enhancement results.
\end{abstract}

Keywords: Rain, fog, haze, fuzzy logic

\section{INTRODUCTION}

Our world is becoming a digital one in a very quick manner. Digital images and video streams are invading our lifestyle over more than one front: from the way we socialize to how we do shopping, not to forget the daily-life augmented reality accessories, to name the least. In weather conditions such as fog, haze and rain visibility is low and navigation is more difficult, dangerous and slow [1]. This is the point where applications such as driver assistance systems come to the rescue. In order for these systems to function the way they supposed to, bad weather conditions such as rain, fog and haze should be accounted for.

Due to the nature of the degradation these phenomena have on the scene, and the mathematically ill-posed problem they pose, many methods attempted to enhance the scene looking from different angles and taking more than one approach. These methods were successful to some degree. These methods can be divided into two main categories non-realtime and real-time methods.

Among the methods that fall under the first category is the Polarization-based visibility improvements suggested by [2, 3]. This meth is making use of the fact that the airlight scattered by the floating particles in the atmosphere e.g. fog, rain, haze etc. is at least partially polarized. To apply this method, two images are taken through a polarizer filter at different angles. By analyzing the images captured, taking into consideration the effects of the polarization of atmospheric scattering, the process is inverted to neutralize the effect of particles on scene. Polarization-based approach requires more than one image of the same scene; it is too slow to be considered in real-time applications. Another method proposed by [4] approaches the problem of enhancement of single image by using information provided by the user interactively, the result of such enhancement is subjective due to the expected variance in the information provided by different users, not to mention the impracticality to apply it in real-time. Reference [5] acquired the depth information of every pixel in the image/frame needed to solve the equation of the light scattering model by employing an interactive registration process to align the image within an existing 3D model. However, the main disadvantage of this approach is that it needs interactive inputs from the user which is not practical in real-time, in addition to the unavailability of 3D model for all sights.

Reference [6] proposed a method based on statistical regularity they observed from studying a large set of hazefree outdoor images. The prior main observation is that in most local patches in haze-free outdoor images there exist some pixels which have very low intensities in at least one color channel [6] .In the hazy image then, and because the airlight is apparent on a dark object, these dark pixels can be used to determine the true airlight.

This method is suitable for real time enhancement and shows very good results. But a soft matting process is needed to visually improve results of the depth map which consumes time, avoiding the soft matting will result in low quality enhancement.

Fuzzy logic-based technique is the approach used by other researchers, like [7] who proposed an algorithm to deweather fog-degraded images. In this method airlight 
estimation is carried out using fuzzy logic followed by color correction to enhance visibility. This method has a lower complexity than conventional physics-based methods, suitable for Real-time applications, but it suffers from distortions that appear in the enhanced image especially at the smooth regions.

Generally, all previous methods require additional information or wouldn't satisfy the real-time constraint. It is impractical to use these techniques to enhance video in realtime, so we need to come up with a new technique that can make use of the simplicity of fuzzy logic and the stability of the dark channel prior in a fast yet robust way.

\section{BACKGROUND}

Visibility or visual range is defined as: "the distance, under daylight conditions, at which the apparent contrast between a specified type of target and its background (horizon sky) becomes just equal to the threshold contrast of an observer..." [8].

Visibility in bad weather is affected by particles floating in the weather. These particles have two effects on light photons passing through them namely absorption and scattering. As the light travels from an object to the observer, the characteristics that will change considerably by the atmosphere particles are color and intensity.

Lots of efforts have been made to measure the particle size and concentration for a variety of conditions. Table (1) below lists some particle sizes in various weather conditions. we can see that due to the small size of the air molecules, relative to the wavelength of visible light, scattering due to these particles is minimum [9]. Particles with relatively larger sizes produce a variety of weather conditions which will be discussed briefly below. Haze: is small, dry particles cannot be seen by the naked eye and sufficiently numerous suspended in the air to give the air an opalescent appearance such as dust, smoke, foliage exudation, volcanic ashes, sea salt and combustion products obscure the clarity of the sky. Haze has the well noticed grayish or bluish effect on the scene.

Table 1. Weather condition and associated PARTICLE types, sizes and concentrations ([9] - ADAPTED FROM

\begin{tabular}{|l|l|l|l|}
\hline Condition & $\begin{array}{l}\text { Particle } \\
\text { Type }\end{array}$ & $\begin{array}{l}\text { Radius( } \\
\boldsymbol{\mu m})\end{array}$ & $\begin{array}{l}\text { Concentrat } \\
\text { ion(cm-3) }\end{array}$ \\
\hline Air & Molecule & $10^{-4}$ & $10^{-4}$ \\
\hline Haze & Aerosol & $10^{-2}-1$ & $10^{3}-10$ \\
\hline Fog & $\begin{array}{l}\text { Water } \\
\text { Droplet }\end{array}$ & $1-10$ & $100-10$ \\
\hline Cloud & $\begin{array}{l}\text { Water } \\
\text { Droplet }\end{array}$ & $1-10$ & $300-10$ \\
\hline Rain & $\begin{array}{l}\text { Water } \\
\text { Drop }\end{array}$ & $10^{2}-10^{4}$ & $10^{2}-10^{5}$ \\
\hline
\end{tabular}

FOG: A visible small water particles (droplets) which are situated at the Earth's surface and reduces horizontal visibility to less than 0.125 kilometer (5/8 mile) and, unlike drizzle, it does not fall to the ground [11].

Cloud: altitude is the main difference between cloud and fog. The cloud forms in higher level compared to the fog which formed at ground level. The clouds are the visible form of liquid droplet or frozen made of water or various chemicals suspended at higher altitudes [9].

Rain: it forms when the water droplets condensed from the water vapor fall under the influence of its weight.

\section{IMAGE OPTICAL SCATTERING MODEL}

The optical scattering model of haze is widely used in computer vision systems and formulated as follows [12]:

$$
\mathrm{Ip}=\mathrm{tp} \cdot \mathrm{Jp}+(1-\mathrm{tp}) \cdot \mathrm{A}
$$

Where $J p$ and $I p$ denote the original scene radiance and the observed radiance at pixel $p$, respectively. $A$ is the ambient light in the atmosphere, referred to as the airlight. Also tp is the transmission at $p$, determined by the distance between the scene point and the camera. Note that the observed radiance $I p$ is the weighted sum of the original scene radiance $\mathrm{Jp}$ attenuated by $t p$ and the airlight $A$ weighted by $(1-t p)$ [9].

Recent work done on real-time enhancement methods impose special case limitations making them rather problem oriented, such as the case with [1] who assume static background, or requires stable objects in the training frames for temporal clustering [13], others assume that moving objects (changing pixels across frames) are minimal [14], while some other methods are meant mainly to enhance dark images and cannot recover information from the shadowed or dark areas of images that had near-black intensities [15], some like [16] focused on finding the visibility distance and other parameters in Koschmieder's model rather than actually enhancing the video. While the method proposed by [6] need a soft matting algorithm to improve the quality of the transmission map needed for the overall enhancement, which consumes a lot of time as stated by [17]: "A component wise analysis of He et al.'s method shows that the processing time predominantly depends on the soft matting algorithm, therefore reducing or even eliminating the processing time of the soft processing would improve the overall dehazing time greatly". Reference [17] eliminated the soft matting step completely assuming the depth map is after all an estimation, so it would be with little effect to exclude this step and depend solely on the rough estimate provided that the patch size is reduced to $4 \times 4$ instead of $15 \times 15$ as used by HE et al. unfortunately, this resulted in chessboard effect on the recovered scene. The existing literature shows that there are still problems with the speed to account for real-time nature of the problem, so there still a need for a robust method to deliver the enhanced output within the time limit and at the same time with an acceptable enhancement. 
In order to address the problems mentioned earlier, it is of a great importance to come up with a new technique to do the overall enhancement in a robust yet fast way to be able to extend to real-time video enhancement recovering the scene from the effect of rain, fog and haze.

\section{RESEARCH METHODOLOGY}

In order to address the problem of the removal of rain, fog and haze from video sequences and achieve the research objectives, an orderly methodology phases are defined as shown in Fig. 1.

\subsection{Problem Identification}

In this phase, the problem of the enhancement of fog, haze and rain video frames is investigated thoroughly by addressing the problem of neutralizing the bad effects of rain, fog and haze within the time limit.

\subsection{Video Acquisition}

In this phase, we will use experimental set-up camera onboard of a vehicle to obtain the foggy and hazy video sequences from different locations and with various levels of visibility in order to apply the proposed methods to remove the effect of bad weather. The captured video sequences will provide input to the suggested system and the system will process the video frames to improve the visibility.

\subsection{Proposed Approach}

After the Analysis of Current Techniques phase, comes this phase which intends to propose methods to address the limitations in existing methods to meet the aim and objectives of the research.

\subsection{Implementation}

Implementing the proposed methods mentioned in the previous phase will be done in this phase. This implementation will be carried out using object oriented programming language such as Java. To fulfill the aim and objectives of the research, different algorithms will be implemented to remove the fog effects and enhance the visibility of the video sequence.

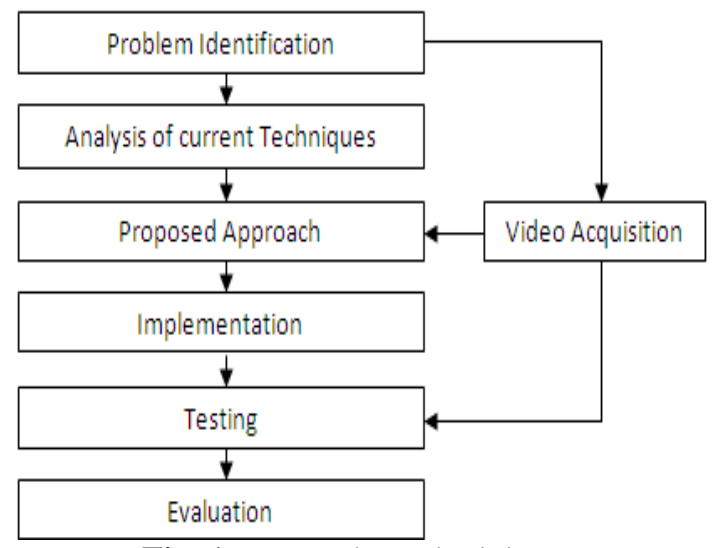

Fig. 1. Research Methodology

\subsection{Testing}

This phase is meant for the necessary checks where the suggested system will be tested. The testing of the system in this phase is considering speed and quality of output video sequence. Then if the system will not be producing the satisfactory results, it will be further improved accordingly.

\subsection{Evaluation}

Results evaluation of the proposed methods is done in this phase, these results are going to be compared with the existing methods in order to examine the speed and the quality of output video. To evaluate results, two methods will be used namely:

\subsection{Subjective Method:}

Absolute Categorical Rating (ACR) will be used, where paired comparisons are used, an observer chooses among two samples the one having greater perceived quality. Raw observer responses to the paired comparison task are converted to scale values. Samples are presented in a random order and use a coarse resolution rating scale for evaluation .Different video sequences with different levels of visibility taken in various weather conditions to exam the quality of output video sequences based on human perception. Number of expert and non-expert users will be invited to examine the quality of the output video sequences, and then their responses will be recorded using a five-degree scale on questionnaires designed especially for this purpose.

\subsection{The Statistical Method:}

In this method, a histogram we will be used to evaluate the enhanced video frames before and after applying the enhancement algorithms. The suggested method will be also compared with the established methods as well as the latest evaluation methods.

\section{REAL-TIME VISIBILITY ENHANCEMENT}

To enhance the video sequence neutralizing the effects of rain, fog and haze from input video frame, atmospheric scattering model is used. Our proposed method consists of three steps:

Fog Effect Map estimation using the dark channel prior, estimation of the airlight $\mathrm{A}$, and finally using the output of the previous steps to enhance the scene luminance for input video frame using fuzzy logic-based technique. These steps are shown in Fig. 2.

\subsection{Estimating the Rough Fog Effect Map}

Using the dark channel prior we can estimate the rough Fog Effect Map over the scene which is calculated as follows: 
The dark channel Idark is defined by He et al. as:

$$
I^{\operatorname{dark}}(x)=\min _{c \in\{r, g, b\}}\left(\min _{y \in \Omega(x)}\left(I^{c}(y)\right)\right)
$$

Where:

$\mathrm{I}^{\mathrm{c}}$ is a colour channel of I the input image

$\Omega(\mathrm{x})$ is a local patch of a certain size centered at $\mathrm{x}$.

\subsection{Estimating Airlight}

A lot of previous methods estimate the airlight A by locating the high intensity pixel in the image [18]. A very bright object with little fog effect can violate this estimation. In order to overcome this problem, we suggest an algorithm to find the airlight $\mathrm{A}$ in which the brightest pixels of the images dark channel is located, then airlight is chosen to be the brightest pixel in the queue provided that it is located in the uppermost area of the image.

\subsection{Enhancing the Overall Scene}

Generally the sky region of the image will be in the upper most area of the image under which resides the most affected area of the image. Pixel values tend to be least affected by fog in the near (lower most) region and vice versa. These properties lead to the definition of two fuzzy input variables namely: Image Region and Pixel Value.

The Image Region is characterized as one of four fuzzy sets, namely Very Near, Near, Little Far and Faraway depending on the fog effect level when projected over the input image. The Pixel Value is characterized as one of Dark, Gray or Bright fuzzy sets. Also we can set the output fuzzy variable. Enhanced Pixel Value which can be characterized as one of the following fuzzy sets Very Dark, Dark, Gray, Bright and Very Bright. Then the membership functions for these fuzzy sets are presumed to be as in Fig. 3, Fig. 4 and Fig. 5 respectively.

fuzzy logic -based technique similar to the one used in [7] will be used in which human intuition is incorporated to make soft decisions on each condition [19], the presumed rules (knowledge base) are as follows:

Rule 1: if (Pixel Value is Gray) then the (Enhanced Pixel Value is Gray). (1)

Rule 2: if (Image Region is Very Near) and (Pixel Value is Dark), then the (Enhanced Pixel Value is Dark).

Rule 3: if (Image Region is Not Very Near) and (Pixel Value is Dark), then the (Enhanced Pixel Value is Very Dark).

Rule 4: if (Image Region is Faraway) and (Pixel Value is Bright), then the (Enhanced Pixel Value is Very Bright).

Rule 5 : if (Image Region is Not Faraway) and (Pixel Value is Bright), then the (Enhanced Pixel Value is Bright).
A Mamdani Fuzzy Inference System (FIS) is used, where the (FIS) exploits the rules above (knowledge base) to infer the enhanced pixel intensity values leading to the overall enhancement of the scene as illustrated in Fig. 6.

Steps of the functional operations in fuzzy inference system are,

\subsection{Fuzzification:}

In this process, membership functions defined on input variables are applied to their actual values to determine the degree of truth for each rule premise or antecedent.

\subsection{Fuzzy Inferencing (Apply Implication Method):}

Truth value for the premise of each rule is computed and applied to the conclusion part of each rule. This results in one fuzzy set to be assigned to each output variable for each rule.

\subsection{Aggregation Of All Outputs:}

It is the process where the outputs of each rule are combined into a single fuzzy set.

\subsection{Defuzzification:}

In this step the fuzzy output set is converted to a crisp number several methods can be applied to get the output crisp value, we will be using the centroid method.

\section{IMPLEMENTATION}

In order to implement the suggested methods and enhance the scene neutralizing the effects of bad weather, the following steps will be performed:

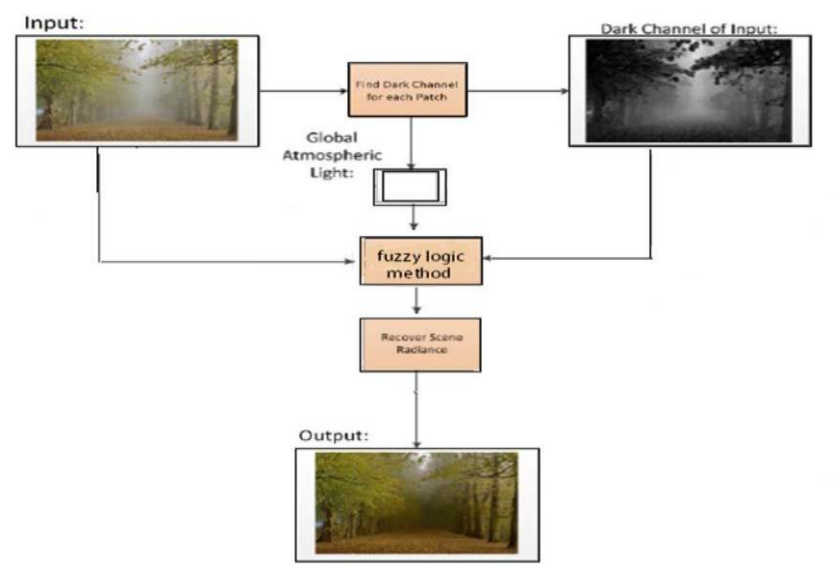

Fig 2 Proposed Method Flow Chart.

\subsection{Acquire Video Sequences in Bad Weather}

We will use on-board mounted experimental camera with high resolution colors to capture video sequences in various bad weather conditions (The readily available image enhancement features in the camera will be turned off). These video sequences will be the input for our suggested system. 


\subsection{Acquire Video Sequences in Clear Weather}

After performing the previous step, the mounted camera will be used again but this time in a clear ideal weather conditions to capture video sequences in similar scenarios to the ones used earlier, this is done for the sake of comparison. This will be the target sample. This video sequence will be used in the testing step.

\subsection{Applying the Suggested Methods}

After the database of video sequences has been created with different type of video sequences captured in different conditions, Estimating airlight and Scene Enhancement methods will be applied to remove the effects of suspended particles from input video frames and will produce higher quality video sequences.

In order to evaluate our proposed methods, we will apply two main tests. First, Absolute Categorical Rating (ACR) will be used where the output video sequences of suggested system will be visually compared (by number of participants) with the video sequences that were captured in clear weather conditions (target video sequences). Second, the quality of output video frames will be statistically compared with input video frames; furthermore, they will be compared with state of the art research methods. The output video sequences should be primarily similar to the video sequences that are captured in a clear day and they should be delivered within the time limit.

\section{DISCUSSION}

Speed is a very important factor in our proposed algorithms, and so we gave a great care to the time limit enforced by the real-time nature of the problem. Soft matting algorithms are time-consuming so we preferred to use fuzzy logic-based technique to map pixel-values to their enhanced values. This is done by utilizing enhancement functions derived from the atmospheric scattering model. Integrating the dark channel prior with a fuzzy logic-based technique based on the stable atmospheric scattering model will yield a fast yet high quality enhancement results, this is because we skipped the timeconsuming soft matting step substituting it with soft decision, fast, fuzzy logic-based technique.

\section{CONCLUSION}

Enhancing any outdoor video sequences that are captured in bad weather is very much affected by the real-time nature of the problem. Faster and more robust method is of a great importance to enhance the scene neutralizing the effects of the different aerosols suspended in the air by using the scattering atmospheric model.

In this research fuzzy logic-based technique is suggested in order to remove the effect of atmospheric particles from video frames. This is done in a fast manner eliminating the need to use time consuming algorithms used to increase the quality of the output, also airlight is estimated from the dark channel pixel values of the input frame enforcing the constraint of the image region.

\section{REFERENCES}

[1] John, Jisha, and M. Wilscy. "Enhancement of weather degraded video sequences using wavelet fusion." In 7th IEEE International Conference on Cybernetic Intelligent Systems, 2008. CIS 2008., pp. 1-6. IEEE, 2008.

[2] Namer, Einav, and Yoav Y. Schechner. "Advanced visibility improvement based on polarization filtered images." In Optics \& Photonics 2005, pp. 588805588805. International Society for Optics and Photonics, 2005.

[3] Schechner, Yoav Y., and Nir Karpel. "Recovery of underwater visibility and structure by polarization analysis." Oceanic Engineering, IEEE Journal of 30, no. 3 (2005): 570-587.

[4] Narasimhan, Srinivasa G., and Shree K. Nayar. "Interactive (de) weathering of an image using physical models." In IEEE Workshop on Color and Photometric Methods in Computer Vision, vol. 6, no. 6.4, p. 1. France, 2003.

[5] Kopf, Johannes, Boris Neubert, Billy Chen, Michael Cohen, Daniel Cohen-Or, Oliver Deussen, Matt Uyttendaele, and Dani Lischinski. "Deep photo: Model-based photograph enhancement and viewing." In ACM Transactions on Graphics (TOG), vol. 27, no. 5, p. 116. ACM, 2008.

[6] He, Kaiming, Jian Sun, and Xiaoou Tang. "Single image haze removal using dark channel prior." Pattern Analysis and Machine Intelligence, IEEE Transactions on 33, no. 12 (2011): 2341-2353.

[7] Desai, Nachiket, Aritra Chatterjee, Shaunak Mishra, Dhaval Chudasama, Sunav Choudhary, and Sudhir Kumar Barai. "A Fuzzy Logic Based Approach to DeWeather Fog-Degraded Images." In Sixth International Conference on Computer Graphics, Imaging and Visualization, 2009. CGIV'09,pp. 383387. IEEE, 2009.

[8] Huschke, Ralph E., and Charles E. Brooks, eds. Glossary of meteorology. Vol. 216. Boston: American Meteorological Society, 1959.

[9] Narasimhan, Srinivasa G., and Shree K. Nayar. "Vision and the atmosphere." International Journal of Computer Vision 48, no. 3 (2002): 233-254.

[10] McCartney, Earl J. "Optics of the Atmosphere: Scattering by molecules and particles." New York, John Wiley and Sons, Inc., 1976. 421 p. 1 (1976).

[11] No, FEDERAL METEOROLOGICAL HANDBOOK. "Surface Weather Observations and Reports." (2005).

[12] Yu, Jing, and Qingmin Liao. "Fast single image fog removal using edge-preserving smoothing." In IEEE International Conference on Acoustics, Speech and Signal Processing (ICASSP), 2011, pp. 1245-1248. IEEE, 2011.

[13] Jia, Zhen, Hongcheng Wang, Rodrigo E. Caballero, Ziyou Xiong, Jianwei Zhao, and Alan Finn. "A twostep approach to see-through bad weather for surveillance video quality enhancement." Machine Vision and Applications 23, no. 6 (2012): 1059-1082. 
[14] Kim, Jin-Hwan, Won-Dong Jang, Yongsup Park, Dong-Hahk Lee, Jae-Young Sim, and Chang-Su Kim. "Temporally x real-time video dehazing." In 19th IEEE International Conference on Image Processing (ICIP), 2012, pp. 969-972. IEEE, 2012.

[15] Rivera, Adin Ramirez, Byungyong Ryu, and Oksam Chae. "Content-Aware Dark Image Enhancement Through Channel Division." Image Processing, IEEE Transactions on 21, no. 9 (2012): 3967-3980.

[16] Hautiere, Nicolas, and Didier Aubert. "Contrast restoration of foggy images through use of an onboard camera." In Intelligent Transportation Systems, 2005. Proceedings. 2005 IEEE, pp. 601-606. IEEE, 2005.

[17] Christian T. Thurow. "Real-Time Image Dehazing." Diploma Thesis, 2011.

[18] Al-Zubaidy, Yaseen, and Rosalina Abdul Salam. "Removal of Atmospheric Particles in Poor Visibility Outdoor Images." TELKOMNIKA Indonesian Journal of Electrical Engineering 11, no. 8 (2013).

[19] Hasikin, Khairunnisa, and Nor Ashidi Mat Isa. "Enhancement of the low contrast image using fuzzy set theory." In UKSim 14th International Conference on Computer Modelling and Simulation (UKSim), 2012, pp. 371-376. IEEE, 2012. 